\title{
The joint association of diabetes distress and depressive symptoms with all-cause mortality in Japanese individuals with type 2 diabetes: a prospective cohort study (Diabetes Distress and Care Registry in Tenri [DDCRT 20])
}

\author{
Yasuaki Hayashino ${ }^{1}$ (D) Shintaro Okamura ${ }^{1} \cdot$ Satoru Tsujii ${ }^{1}$ (D) Hitoshi Ishii ${ }^{2}$ (D) . for the Diabetes Distress and Care \\ Registry at Tenri Study Group
}

Received: 28 July 2020 / Accepted: 31 July 2020 / Published online: 19 September 2020

(C) Springer-Verlag GmbH Germany, part of Springer Nature 2020

\begin{abstract}
Aims/hypothesis The independent association of depressive symptoms and diabetes distress with mortality risk in individuals with diabetes has not been evaluated. We examined the temporal joint association of diabetes distress and depressive symptoms at baseline and the subsequent risk of all-cause mortality.

Methods The longitudinal data of 3118 individuals with type 2 diabetes were obtained from a large Japanese diabetes registry. To assess the joint association of diabetes distress and depressive symptoms at baseline with the subsequent risk of all-cause mortality, the Cox proportional hazards model was used with adjustment for potential confounders.

Results The mean age, $\mathrm{BMI}$ and $\mathrm{HbA}_{1 \mathrm{c}}$ levels were 64.7 years, $24.6 \mathrm{~kg} / \mathrm{m}^{2}$ and $58.6 \mathrm{mmol} / \mathrm{mol}(7.5 \%)$, respectively, and $38.1 \%$ of the participants were women. In the multivariable-adjusted models evaluating the diabetes distress and depressive symptoms separately, the HRs for all-cause mortality were 1.67 (95\% CI 1.14, 2.43; $p=0.008)$ and 1.40 (95\% CI 1.05, 1.85; $p=0.020)$, respectively. In such models evaluating the joint association of diabetes distress and depressive symptoms, compared with individuals without diabetes distress or depressive symptoms ( $\mathrm{DD}^{-} / \mathrm{DS}^{-}$), the HRs for all-cause mortality for the group without diabetes distress but with depressive symptoms $\left(\mathrm{DD}^{-} / \mathrm{DS}^{+}\right)$, with diabetes distress but without depressive symptoms $\left(\mathrm{DD}^{+} / \mathrm{DS}^{-}\right)$, and with diabetes distress and depressive symptoms $\left(\mathrm{DD}^{+} / \mathrm{DS}^{+}\right)$were $1.34(95 \%$ CI 0.99, 1.86; $p=0.056), 1.96(95 \%$ CI 1.10, 3.50; $p=0.023)$ and 1.71 (95\% CI 1.06, $2.77 ; p=$ $0.029)$, respectively. We did not observe a significant interaction between diabetes distress and depressive symptoms with all-cause mortality risk $(p=0.2636)$. In the stratified analysis by sex, a significant joint association of diabetes distress and depressive symptoms with the risk of all-cause mortality was observed only in men.

Conclusions/interpretation Diabetes distress and depressive symptoms were independently associated with all-cause mortality risk in male participants with type 2 diabetes, but we did not observe a significant interaction between diabetes distress and depressive symptoms in relation to all-cause mortality.
\end{abstract}

Keywords Depressive symptom $\cdot$ Diabetes distress $\cdot$ Epidemiology $\cdot$ Human $\cdot$ Mortality risk $\cdot$ Patient-reported outcome $\cdot$ Type 2 diabetes

Members of the Diabetes Distress and Care Registry at Tenri Study Group are listed in the Appendix.

Yasuaki Hayashino

hayasino-y@umin.net

Department of Endocrinology, Tenri Hospital, Tenri, Nara, Japan

2 Department of Diabetology, Nara Medical University, Kashihara, Nara, Japan

\section{Abbreviations}

CES-D Center for Epidemiologic Studies Depression

CRC Clinical research coordinator

$\mathrm{DD}^{-} / \mathrm{DS}^{-}$Without diabetes distress or depressive symptoms

$\mathrm{DD}^{-} / \mathrm{DS}^{+} \quad$ Without diabetes distress but with depressive symptoms

$\mathrm{DD}^{+} / \mathrm{DS}^{-} \quad$ With diabetes distress but without depressive symptoms 


\section{Research in context}

What is already known about this subject?

- Many people with diabetes feel burdened by the never-ending challenge of self-management

- Depressive symptoms and diabetes distress have been associated with high risk of mortality

What is the key question?

- Are high levels of diabetes distress and depressive symptoms jointly associated with high risk of mortality?

What are the new findings?

- High levels of diabetes distress and depressive symptoms were independently associated with higher risk of allcause mortality in male participants with type 2 diabetes, but we did not observe a significant interaction between diabetes distress and depressive symptoms in relation to risk of all-cause mortality

How might this impact on clinical practice in the foreseeable future?

- These findings provide new evidence for the importance of managing diabetes distress and depressive symptoms as part of diabetes care

\section{$\mathrm{DD}^{+} / \mathrm{DS}^{+} \quad$ With diabetes distress and depressive symptoms \\ PAID Problem Areas in Diabetes \\ UACR Urinary albumin/creatinine ratio}

\section{Introduction}

Over the past few decades, the prevalence of type 2 diabetes has dramatically increased worldwide [1], with projections of even greater growth over the coming decades [2]. The high prevalence of type 2 diabetes, along with type 2 diabetes complications, tremendously contribute to the burden of mortality and disability globally. The diabetes epidemic is estimated to cause more than 4.2 million deaths annually in 2019 [3].

Self-management is the basis of diabetes care, and people with diabetes are expected to carry out daily self-management activities to help prevent diabetes-related morbidity and mortality risk, but this is more difficult than it appears. Individuals with diabetes often experience diabetes distress, and depression is also common in people with diabetes $[4,5]$. Both diabetes distress and depressive symptoms can interfere with self-care activities in individuals with diabetes, including physical activity, healthy food intake, medication adherence and smoking cessation [6-9]. Diabetes distress reflects the person's emotional response to the burden of living with a largely self-managed chronic disease and its debilitating complications [10], and depression is a mood disorder defined not by cause but as a syndromal diagnosis. About 10-30\% of individuals with diabetes suffer from depression [11-14] and about 20\% suffer from diabetes distress [11, 13-17]. Recently, it has been reported that both diabetes distress and depression were separately associated with diabetic complications and mortality risk (although they were not evaluated simultaneously) [18, 19]. Although depression and diabetes distress are different constructs, as the distress results from the emotional burden that a person feels from living with diabetes, the constructs overlap to some extent, and about $5 \%$ of people with diabetes have both depression and diabetes distress. The association of depression and diabetes distress with mortality risk in individuals with diabetes needs to be examined. However, diagnosis of clinical depression requires substantial time and cost, and it is sometimes difficult to diagnose clinical depression in a large epidemiological study. Thus, in many epidemiological studies, standardised instruments designed to evaluate the presence or severity of self-reported depressive symptoms are used [20]. In general, these instruments measure depressive symptomatology rather than clinical depression. The Center for Epidemiologic Studies Depression (CES-D) scale is among the most widely used questionnaires for measuring depressive symptoms in epidemiological studies [21, 22].

In this study, we thus aimed to examine a cohort of Japanese individuals with type 2 diabetes from a large-scale Japanese diabetes registry to determine prospectively the joint association of diabetes distress and depressive symptoms measured by the CES-D scale at baseline with the subsequent risk of all-cause mortality risk.

\section{Methods}

Participants Patient data were derived from a diabetes registry at Tenri Hospital, a regional tertiary-care teaching hospital in Japan. From October 2009 to August 2010, the registry recruited individuals diagnosed with diabetes who visited the outpatient clinic [23-25]. This study included 1 additional year of follow-up to the data in our previous report that evaluated the association between 
diabetes distress and mortality risk [19]. Individuals diagnosed with impaired glucose tolerance and/or impaired fasting glucose using an OGTT were excluded. A total of 5133 individuals with diabetes visited the hospital, of which 81 were deemed as unable to complete the survey (as assessed by an attending physician) because of dementia $(n=15)$, mental retardation $(n=6)$, schizophrenia $(n=3)$, severe major depression $(n=2)$, low vision $(n=$ $35)$, poor general condition $(n=3)$ and other comorbidities $(n=$ 17); thus, these individuals were not included in our study. Of the 5052 eligible patients, $3898(77.2 \%)$ consented to participate in the study, and these participants were given a self-administered survey form at registration. In addition, we excluded individuals with diabetes other than type 2 diabetes $(n=199)$, those with a past medical history of any malignant neoplasms $(n=365)$, and those who did not complete the Problem Areas in Diabetes (PAID) survey or CES-D scale $(n=216)$. The data of the remaining 3118 patients were used for the current analysis. The participants completed the form with the assistance of clinical research coordinators (CRCs). The Ethics Committee of the Tenri Hospital approved this study.

Data collection At baseline and every year thereafter, trained CRCs collected participant demographics from medical charts, which included age, sex, body weight, past medical history (e.g. micro- and macrovascular complications) and treatment modalities. Prescription data were obtained electronically from the drug ordering system. Participants underwent laboratory tests at the baseline visit, which included random measurements (fasting was not required) such as $\mathrm{HbA}_{1 \mathrm{c}}$ and urinary albumin/ creatinine ratio (UACR). $\mathrm{HbA}_{1 \mathrm{c}}$ levels were expressed in accordance with the National Glycohemoglobin Standardization Program (NGSP) guidelines, as recommended by the Japan Diabetes Society [26]. On the same day as the laboratory test, participants completed the self-administered survey including the PAID and CES-D. Two trained CRCs independently input data from the medical charts or from written data to the data server at the Data Management Centre of Tenri hospital, and rechecked the medical charts and original survey sheets for any inconsistency in data.

Assessment of diabetes distress and depressive symptoms Participants completed the Japanese version of the PAID questionnaire embedded in the self-administered survey at baseline. Developed by Polonsky et al, the PAID survey is a 20 -item selfreport measure of diabetes-related emotional distress with high internal reliability and clinical utility. It was translated into Japanese by Ishii, and the Japanese version shows good internal consistency and validity $[27,28]$. The questions are framed as follows: 'From your own perspective, to what degree are the following diabetes-related issues currently a problem for you?', with a 5-point scale to rate each item (ranging from 0 , 'not a problem', to 4, 'a serious problem'). According to the recommendation of the authors of the PAID measure, a total score was computed by summing the total item responses and multiplying by 1.25 to produce a total score that ranges from 0 to 100 . The known cut-off point to define diabetes distress by the total PAID score is 40 or higher; this has been found to be around 1 SD above the mean across different studies and has discriminative validity [28, 29].

Depressive symptoms were assessed using the Japanese version of CES-D, which was translated from the original version [21] and validated in the Japanese language [22]. The instrument consists of 20 items, using a four-point Likert scale. The possible range of scores is $0-60$, and a cutoff score of $\geq 16$ commonly indicates clinically significant depressive symptoms [30].

Mortality Every year, researchers listed the participants who were registered at baseline but later dropped out of the survey, and the vital status of each of these participants was checked using the information on medical charts. If a participant was no longer attending our hospital and the vital status was unknown, we conducted a follow-up postal and telephone survey. For participants who had died, the underlying cause of death was classified on the basis of the review of inpatient and outpatient medical records just preceding the death, death certificate and other records (e.g. autopsy report) by the methods used in the Action to Control Cardiovascular Risk in Diabetes (ACCORD) study group [31]. A death certificate diagnosis was used when no other records were available. Two physicians reviewed all the deaths and determined the underlying cause of death by consensus.

Statistical analysis Continuous variables are expressed as mean and SD or IQR, where indicated. Intergroup differences were evaluated using unpaired Student's $t$ test and the $\chi^{2}$ analysis for categorical variables.

First, we evaluated the joint association of diabetes distress and depressive symptoms with all-cause mortality risk by constructing four categories of the combination of diabetes distress and depressive symptoms: (1) without diabetes distress or depressive symptoms $\left(\mathrm{DD}^{-} / \mathrm{DS}^{-}\right)$; (2) without diabetes distress but with depressive symptoms $\left(\mathrm{DD}^{-} / \mathrm{DS}^{+}\right)$; (3) with diabetes distress but without depressive symptoms $\left(\mathrm{DD}^{+} / \mathrm{DS}^{-}\right)$; and (4) with diabetes distress and depressive symptoms $\left(\mathrm{DD}^{+} / \mathrm{DS}^{+}\right)$. Person-time was calculated from the date of the survey at baseline to date of death or the last survey, whichever occurred first. Two statistical models were used: the first model was adjusted for age and sex; the second model was adjusted for multiple variables, including age, sex, BMI, $\mathrm{HbA}_{1 \mathrm{c}}$, type of diabetes therapy (diet only, oral medication only, insulin or glucagon-like receptor agonist injection), UACR ( $<3 \mathrm{mg} / \mathrm{mmol}, 3$ to $<30 \mathrm{mg} / \mathrm{mmol}, \geq 30 \mathrm{mg} / \mathrm{mmol}$ ), history of any diabetic retinopathy, symptomatic diabetic neuropathy and past medical history (acute myocardial infarction, chronic stable angina, peripheral artery disease, leg 
ulceration, ischaemic stroke, haemorrhagic stroke). The Cox proportional hazards model was used to estimate the HRs and 95\% CIs for all-cause mortality for participants in the $\mathrm{DD}^{-} /$ $\mathrm{DS}^{+}, \mathrm{DD}^{+} / \mathrm{DS}^{-}$and $\mathrm{DD}^{+} / \mathrm{DS}^{+}$categories, using the $\mathrm{DD}^{-} / \mathrm{DS}^{-}$ group as a reference.

Second, we evaluated the association between each of the PAID ( $\geq 40$ vs $<40)$ and CES-D $(\geq 16$ vs $<16)$ total score categories and risk of all-cause mortality. The two previously mentioned statistical models (age- and sex-adjusted or multivariable-adjusted Cox proportional hazards model) were used to estimate the HRs and 95\% CIs for all-cause mortality for diabetes distress or depressive symptoms.

Finally, we replicated all the aforementioned analyses stratified by sex, as we previously reported that the association between diabetes distress and all-cause mortality risk was observed only in male participants [19]. Statistical interactions were analysed using likelihood ratio tests, which compared the $-2 \log$ (likelihood) between two nested models: one considering the main effects alone and the other considering the main effects and additional interaction terms. Causespecific death analysis was not performed because of the very small number of outcomes in each death category. All reported $p$ values are two-sided, and $p<0.05$ was regarded as statistically significant, other than for the test for interaction $(p<0.1)$. All analyses were performed using Stata/SE version 15.1 (Stata Corporation, USA).

\section{Results}

The mean (SD) age, BMI and $\mathrm{HbA}_{1 \mathrm{c}}$ level were 64.7 years (11.3), $24.6 \mathrm{~kg} / \mathrm{m}^{2}$ (3.9), and $58.6 \mathrm{mmol} / \mathrm{mol}$ (12.6) $(7.5 \%$ [1.1]), respectively (Table 1). Diabetes distress was significantly associated with younger age $(p=0.001)$, female sex $(p<0.001)$, poorer glycaemic control $(p<0.001)$, higher use of injection therapy $(p<0.001)$ and higher prevalence of symptomatic neuropathy $(p<0.001)$, while depressive symptoms were significantly associated with female sex $(p=$ $0.005)$, higher use of injection therapy $(p=0.004)$, higher prevalence of symptomatic neuropathy $(p<0.001)$, past history of myocardial infarction $(p=0.001)$, and peripheral artery disease $(p=0.005)$. We observed 238 cases of any death during the median follow-up of 7.1 years (incidence ratio $=$ 12.3 per 1000 person-years for all participants [Table 2], 14.6 per 1000 person-years for male participants, 8.6 per 1000 person-years for female participants). The most frequent cause of death was malignant neoplasia (26.5\%), followed by CVD $(20.6 \%)$, infection (12.2\%), and ischaemic or haemorrhagic stroke $(5.0 \%)$. This order of ranking was similar to the results of the survey on the causes of death in 45,708 Japanese individuals with diabetes during the years 2001-2010 published by the Japan Diabetes Society [32].
The incidence ratios of death for those with PAID scores $<40$ and $\geq 40$ were 12.0 and 14.8 per 1000 person-years, respectively (Table 2), and the incidence ratios of death for those with CES-D scores $<16$ and $\geq 16$ were 11.0 and 15.7 per 1000 person-years, respectively. The results of the joint association of diabetes distress and depressive symptoms with all-cause mortality risk are shown in Table 3. Compared with the $\mathrm{DD}^{-} /$ $\mathrm{DS}^{-}$group, the age- and sex-adjusted HRs for all-cause mortality for the $\mathrm{DD}^{-} / \mathrm{DS}^{+}, \mathrm{DD}^{+} / \mathrm{DS}^{-}$and $\mathrm{DD}^{+} / \mathrm{DS}^{+}$were $1.49(95 \%$ CI 1.10, 2.00; $p=0.009) ; 1.80$ (95\% CI 1.02, 3.18; $p=0.043$ ); and 1.70 (95\% CI 1.07, 2.72; $p=0.026)$, respectively. After adjusting for possible confounders, the HR for those in the $\mathrm{DD}^{-} / \mathrm{DS}^{+}$group was within the non-significant range (HR 1.34 [95\% CI $0.99,1.86] ; p=0.056$ ). Moreover, after stratifying by sex, we observed a significant association between the combination of diabetes distress and depressive symptoms categories and all-cause mortality risk only in men. In male participants, the HRs for all-cause mortality for those in the $\mathrm{DD}^{-} /$ $\mathrm{DS}^{+}, \mathrm{DD}^{+} / \mathrm{DS}^{-}$and $\mathrm{DD}^{+} / \mathrm{DS}^{+}$groups were $1.44(95 \% \mathrm{CI} 1.00$, $2.07 ; p=0.049) ; 2.45$ (95\% CI 1.27, 4.75; $p=0.008)$; and 2.26 (95\% CI 1.32, 3.88; $p=0.003$ ), respectively.

Compared with participants without diabetes distress, the age- and sex-adjusted HR for all-cause mortality was 1.57 (95\% CI 1.08, 2.27; $p=0.017$ ) for those with diabetes distress (Table 4). Adjustment for possible confounders slightly strengthened the association; the multivariable-adjusted HR for all-cause mortality was 1.67 (95\% CI 1.14, 2.43; $p=$ 0.008 ). Stratified by sex, we observed a significant association between diabetes distress and all-cause mortality risk only in men. The multivariable-adjusted HRs for all-cause mortality for those with diabetes distress were 2.11 (95\% CI 1.38, 3.23; $p=0.001)$ in male participants and 0.91 (95\% CI 0.41, 2.01; $p=0.813)$ in female participants with a statistically significant interaction ( $p$ value for interaction $=0.0524$ ).

Compared with participants without depressive symptoms, the age- and sex-adjusted HR for all-cause mortality was 1.47 (95\% CI 1.13, 1.93; $p=0.004$ ) for those with depressive symptoms (Table 4). Adjustment for possible confounders slightly attenuated the association; the multivariable-adjusted HR for all-cause mortality was 1.40 (95\% CI 1.05, $1.85 ; p=0.020$ ). Additionally, after stratifying by sex, we observed a significant association between depressive symptoms and all-cause mortality risk only in men. The multivariable-adjusted HRs for all-cause mortality for those with depressive symptoms were 1.52 (95\% CI $1.10,2.10 ; p=0.011)$ in male participants and $1.23(95 \% \mathrm{CI}$ $0.73,2.06 ; p=0.431$ ) in female participants without a statistically significant interaction ( $p$ value for interaction $=0.3123$ ).

\section{Discussion}

To our knowledge, this is the first study that prospectively evaluated the joint association of diabetes distress and 
Table 1 Participant characteristics based on PAID survey score category $(<40, \geq 40)$ or CES-D score category $(<16, \geq 16)$ at baseline

\begin{tabular}{|c|c|c|c|c|c|c|c|c|}
\hline \multirow[t]{2}{*}{ Variable } & \multicolumn{4}{|c|}{ PAID total score } & \multicolumn{4}{|c|}{ CES-D total score } \\
\hline & $\begin{array}{l}\text { All } \\
3118\end{array}$ & $\begin{array}{l}<40 \\
2747\end{array}$ & $\begin{array}{l}\geq 40 \\
371\end{array}$ & $p$ value & $\begin{array}{l}\text { All } \\
3118\end{array}$ & $\begin{array}{l}<16 \\
2256\end{array}$ & $\begin{array}{l}\geq 16 \\
862\end{array}$ & $p$ value \\
\hline PAID score & $29(23-40)$ & $27(22-36)$ & $60(55-66)$ & & $12.9 \pm 7.9$ & $9 \pm 4.0$ & $22.8 \pm 6.9$ & \\
\hline Age, years & $64.7(11.3)$ & $65.0(11.1)$ & $62.4(12.5)$ & 0.001 & $64.7 \pm 11.3$ & $64.8 \pm 10.9$ & $64.4 \pm 12.1$ & 0.362 \\
\hline Female, $\%$ & 38.1 & 36.4 & 50.9 & $<0.001$ & 38.1 & 36.6 & 42.1 & 0.005 \\
\hline BMI, $\mathrm{kg} / \mathrm{m}^{2}$ & $24.6(3.9)$ & $24.6(3.9)$ & $24.9(4.2)$ & 0.062 & $24.6 \pm 3.9$ & $24.6 \pm 3.8$ & $24.7 \pm 4.1$ & 0.409 \\
\hline $\mathrm{HbA}_{1 \mathrm{c}}$ & & & & $<0.001$ & & & & 0.180 \\
\hline IFCC (mmol/mol) & $58.6(12.6)$ & $58.1(12.2)$ & $62.8(15.0)$ & & $58.6 \pm 12.6$ & $58.4 \pm 12.3$ & $59.1 \pm 13.5$ & \\
\hline NGSP $(\%)$ & $7.5(1.1)$ & $7.5(1.1)$ & $7.9(1.4)$ & & $7.5 \pm 1.1$ & $7.5 \pm 1.1$ & $7.6 \pm 1.2$ & \\
\hline Type of diabetes therapy, $\%$ & & & & $<0.001$ & & & & 0.004 \\
\hline Diet only & 62.0 & 63.6 & 49.9 & & 62.0 & 63.7 & 57.4 & \\
\hline Oral medication without injection $t x$ & 14.8 & 14.0 & 21.3 & & 14.8 & 14.5 & 15.9 & \\
\hline Insulin or GLP-1RA & 23.2 & 22.4 & 28.8 & & 23.2 & 21.8 & 26.7 & \\
\hline UACR (mg/mmol), \% & & & & 0.593 & & & & 0.492 \\
\hline$<3$ & 50.6 & 50.5 & 51.0 & & 50.6 & 51.1 & 49.3 & \\
\hline 3 to $<30$ & 37.0 & 37.3 & 34.7 & & 37.0 & 37.1 & 36.8 & \\
\hline$>30$ & 12.4 & 12.2 & 14.3 & & 12.4 & 11.9 & 13.9 & \\
\hline Any diabetic retinopathy, $\%$ & 45.2 & 44.6 & 49.1 & 0.309 & 45.2 & 44.4 & 47.4 & 0.353 \\
\hline Symptomatic diabetic neuropathy, $\%$ & 31.0 & 29.7 & 40.4 & $<0.001$ & 31.0 & 27.0 & 41.3 & $<0.001$ \\
\hline \multicolumn{9}{|l|}{ Past medical history, $\%$} \\
\hline Acute myocardial infarction & 9.5 & 9.4 & 10.5 & 0.452 & 9.5 & 8.4 & 12.4 & 0.001 \\
\hline Chronic stable angina & 11.6 & 11.7 & 11.1 & 0.796 & 11.6 & 11.0 & 13.2 & 0.087 \\
\hline Peripheral artery disease & 2.1 & 2.1 & 2.2 & 0.847 & 2.1 & 1.6 & 3.3 & 0.005 \\
\hline Leg ulceration & 1.0 & 1.0 & 0.8 & 1.000 & 1.0 & 0.9 & 1.3 & 0.326 \\
\hline Ischaemic stroke & 8.0 & 8.3 & 5.9 & 0.126 & 8.0 & 7.5 & 9.4 & 0.079 \\
\hline Haemorrhagic stroke & 1.2 & 1.2 & 0.8 & 0.615 & 1.2 & 1.1 & 1.5 & 0.305 \\
\hline
\end{tabular}

Data are median (IQR), means \pm SD or \%

GLP-1RA, glucagon-like peptide-1 receptor agonist; IFCC, International Federation of Clinical Chemistry; NGSP, National Glycohemoglobin Standardization Program; tx, therapy

depressive symptoms with the subsequent risk of all-cause mortality in individuals with type 2 diabetes. Compared with individuals in the $\mathrm{DD}^{-} / \mathrm{DS}^{-}$group, for those in the $\mathrm{DD}^{-} / \mathrm{DS}^{+}$, $\mathrm{DD}^{+} / \mathrm{DS}^{-}$and $\mathrm{DD}^{+} / \mathrm{DS}^{+}$groups at baseline there was an association with higher risk of all-cause mortality risk only in male participants. We did not observe a significant interaction between diabetes distress and depressive symptoms and allcause mortality risk.

Depressive symptoms and diabetes distress are common among individuals with diabetes; about $30 \%$ have depressive
Table 2 PAID survey score $(<40$, $\geq 40)$, CES-D score $(<16, \geq 16)$ and subsequent risk of all-cause mortality

\begin{tabular}{|c|c|c|c|c|}
\hline & Participants, $n$ & Person-years & Deaths, $n$ & Incidence ratio $(95 \% \mathrm{CI})^{\mathrm{a}}$ \\
\hline \multicolumn{5}{|c|}{ PAID total score } \\
\hline$<40$ & 2747 & 17,118 & 205 & $12.0(10.4,13.7)$ \\
\hline$\geq 40$ & 371 & 2223 & 33 & $14.8(10.6,20.9)$ \\
\hline \multicolumn{5}{|c|}{ CES-D total score } \\
\hline$<16$ & 2256 & 14,127 & 156 & $11.0(9.4,12.9)$ \\
\hline$\geq 16$ & 862 & 5214 & 82 & $15.7(12.7,19.5)$ \\
\hline All & 3118 & 19,341 & 238 & $12.3(10.8,14.0)$ \\
\hline
\end{tabular}

${ }^{\text {a }}$ Per 1000 person-years 
Table 3 The joint association of diabetes distress and depressive symptoms with all-cause mortality risk

Diabetes distress and depressive symptoms status

\begin{tabular}{|c|c|c|c|c|c|}
\hline \multirow[b]{2}{*}{ Statistical model } & & \multirow[b]{2}{*}{ iliteraction } \\
\hline & $\mathrm{DD}^{-} / \mathrm{DS}^{-}$ & $\mathrm{DD}^{-} / \mathrm{DS}^{+}$ & $\mathrm{DD}^{+} / \mathrm{DS}^{-}$ & $\mathrm{DD}^{+} / \mathrm{DS}^{+}$ & \\
\hline All participants & $n=2117$ & $n=630$ & $n=139$ & $n=232$ & \\
\hline Age- and sex-adjusted model & Ref. & $1.49(1.10,2.00) ; p=0.009$ & $1.80(1.02,3.18) ; p=0.043$ & $1.70(1.07,2.72) ; p=0.026$ & 0.2504 \\
\hline Multivariable-adjusted model ${ }^{\mathrm{b}}$ & Ref. & $1.34(0.99,1.86) ; p=0.056$ & $1.96(1.10,3.50) ; p=0.023$ & $1.71(1.06,2.77) ; p=0.029$ & 0.2636 \\
\hline Stratified by sex & & & & & $0.2706^{\mathrm{c}}$ \\
\hline Male & $n=1362$ & $n=385$ & $n=68$ & $n=114$ & \\
\hline Multivariable-adjusted model $^{\mathrm{d}}$ & Ref. & $1.44(1.00,2.07) ; p=0.049$ & $2.45(1.27,4.75) ; p=0.008$ & $2.26(1.32,3.88) ; p=0.003$ & 0.3034 \\
\hline Female & $n=755$ & $n=245$ & $n=71$ & $n=118$ & \\
\hline Multivariable-adjusted model $^{\mathrm{d}}$ & Ref. & $1.15(0.63,2.11) ; p=0.653$ & $1.07(0.33,3.50) ; p=0.911$ & $0.90(0.32,2.54) ; p=0.846$ & 0.7356 \\
\hline
\end{tabular}

Data are presented as HR $(95 \% \mathrm{CI})$

${ }^{\mathrm{a}} p$ values for statistical interaction between diabetes distress and depressive symptoms

${ }^{\mathrm{b}}$ Adjusted for age, sex, BMI, $\mathrm{HbA}_{1 \mathrm{c}}$, type of diabetes therapy, UACR, history of any diabetic retinopathy, symptomatic diabetic neuropathy and past medical history (acute myocardial infarction, chronic stable angina, peripheral artery disease, leg ulceration, ischaemic stroke, haemorrhagic stroke)

${ }^{\mathrm{c}} p$ value for statistical interaction between sex and combination category of diabetes distress and depressive symptoms

${ }^{\mathrm{d}}$ Adjusted for age, BMI, $\mathrm{HbA}_{1 \mathrm{c}}$, type of diabetes therapy, UACR, history of any diabetic retinopathy, symptomatic diabetic neuropathy and past medical history (acute myocardial infarction, chronic stable angina, peripheral artery disease, leg ulceration, ischaemic stroke, haemorrhagic stroke)

Ref., reference

symptoms, and $20 \%$ have diabetes distress [16, 17, 33-35]. Not only do these conditions have high prevalence, but they are also associated with the future risk of mortality. Among a cohort of 4184 patients with diabetes at the Group Health Cooperative in Washington State, depressive symptoms assessed using the Patient Health Questionnaire (PHQ-9) at baseline were associated with a significantly higher risk of all- cause mortality (HR for all-cause mortality 2.26 [95\% CI $1.79,2.85]$ ] [36], which is consistent with the results of other studies [37-39]. Only one study, from our research group, has shown the association between diabetes distress and mortality risk [19]. In this study, diabetes distress, classified as a PAID total score $\geq 40$, was associated with a subsequent risk of allcause mortality risk (HR 1.56 [95\% CI 1.17, 2.08]) over

Table 4 The association between diabetes distress by PAID or depressive symptoms by CES-D and the subsequent risk of all-cause mortality

\begin{tabular}{|c|c|c|c|c|c|c|}
\hline \multirow[t]{2}{*}{ Statistical model } & \multicolumn{3}{|c|}{ PAID total score category } & \multicolumn{3}{|c|}{ CES-D total score category } \\
\hline & $<40$ & $\geq 40$ & $\begin{array}{l}p \text { value for } \\
\text { interaction }^{\text {a }}\end{array}$ & $<16$ & $\geq 16$ & $\begin{array}{l}p \text { value for } \\
\text { interaction }\end{array}$ \\
\hline \multicolumn{7}{|l|}{ All participants } \\
\hline$n$ & $n=2747$ & $n=371$ & & $n=2256$ & $n=862$ & \\
\hline Age- and sex-adjusted model & Ref. & $1.57(1.08,2.27) ; p=0.017$ & & Ref. & $1.47(1.13,1.93) ; p=0.004$ & \\
\hline Multivariable-adjusted model $^{\mathrm{b}}$ & Ref. & $1.67(1.14,2.43) ; p=0.008$ & & Ref. & $1.40(1.05,1.85) ; p=0.020$ & \\
\hline \multicolumn{7}{|l|}{ Stratified by sex } \\
\hline Male, $n$ & $n=1747$ & $n=182$ & 0.0524 & $n=1430$ & $n=499$ & 0.3123 \\
\hline Multivariable-adjusted model ${ }^{\mathrm{c}}$ & Ref. & $2.11(1.38,3.23) ; p=0.001$ & & Ref. & $1.52(1.10,2.10) ; p=0.011$ & \\
\hline Female & $n=1000$ & $n=189$ & & $n=826$ & $n=363$ & \\
\hline Multivariable-adjusted model ${ }^{\mathrm{c}}$ & Ref. & $0.91(0.41,2.01) ; p=0.813$ & & Ref. & $1.23(0.73,2.06) ; p=0.431$ & \\
\hline
\end{tabular}

Data are presented as HR $(95 \% \mathrm{CI})$

${ }^{a} p$ values for statistical interaction between sex and diabetes distress or depressive symptoms

${ }^{\mathrm{b}}$ Adjusted for age, sex, BMI, $\mathrm{HbA}_{1 \mathrm{c}}$, type of diabetes therapy, UACR, history of any diabetic retinopathy, symptomatic diabetic neuropathy and past medical history (acute myocardial infarction, chronic stable angina, peripheral artery disease, leg ulceration, ischaemic stroke, haemorrhagic stroke)

${ }^{\mathrm{c}}$ Adjusted for age, BMI, $\mathrm{HbA}_{1 \mathrm{c}}$, type of diabetes therapy, UACR, history of any diabetic retinopathy, symptomatic diabetic neuropathy and past medical history (acute myocardial infarction, chronic stable angina, peripheral artery disease, leg ulceration, ischaemic stroke, haemorrhagic stroke) 
6.1 years of follow-up, even after adjusting for possible confounders, in 3305 individuals with type 2 diabetes. In this research, depressive symptoms were not included in the multivariable-adjusted model because this construct is different from that of diabetes [40].

In the subgroup analysis, the association of diabetes distress or depressive symptoms with all-cause mortality risk was significantly observed only in men, not in women. A previous meta-analysis evaluated whether excess mortality risk associated with depressive symptoms was higher in men than in women, although this study was not limited to the population with diabetes [41]. In the 41,331 participants from 13 studies, depressive symptoms were associated with a higher mortality risk in both women (RR 1.55 [95\% CI $1.32,1.82]$ ) and men (RR 2.04 [95\% CI 1.76, 2.37]), with a significant interaction being observed $(p=0.015)$, indicating a higher risk of mortality in men than women. Compared with women with depressive symptoms, men with depressive symptoms had a 1.97 -fold higher risk of mortality $(95 \% \mathrm{CI}$ $1.63,2.37)$. In several other studies not included in the aforementioned meta-analysis, depressive symptoms were associated with all-cause mortality risk only in men [42, 43]. This could be partly explained by the higher suicide rate in men than in women with major depression [44], surveyed by the National Institute of Mental Health Diagnostic Interview Schedule [45], but whether the high suicide risk of depression is applicable to male individuals with diabetes distress remains unclear to date.

The association between diabetes distress and mortality risk could be speculated to be caused by poor glycaemic control or high risk of complications. In fact, our study has shown that participants with PAID score $\geq 40$ have statistically poorer glycaemic control or higher prevalence of diabetic neuropathy. However, findings from previous research that examined the link between diabetes distress and glycaemic control or complications are mixed. In 3489 individuals with diabetes (type 1 and type 2) in Japan, $\mathrm{HbA}_{1 \mathrm{c}}$ levels were higher in participants with diabetes distress measured by PAID than in those without [14]. A similar association was observed in other countries [35, 46,47], and when using a different tool to measure diabetes distress (the Diabetes Distress Scale) [46]; however, these studies were performed in a cross-sectional manner, and it could not be said that diabetes distress causes poor glycaemic control. Although, a recently published article reported that diabetes distress (PAID score $\geq 40$ ) was not prospectively linked to elevated $\mathrm{HbA}_{1 \mathrm{c}}$ levels in individuals with type 2 diabetes [48]; however, a limitation of this study is the short duration of follow-up (2 years). A PAID-5 score (which is evaluated using 5 from 20 items of PAID) $\geq 40$ was associated with a higher prevalence of microand macrovascular complications in a cross-sectional study of 2374 participants with type 2 diabetes [47]. However, the cross-sectional nature of the study design may limit the interpretation of the direction of causality. Moreover, two research studies have examined the prospective association between distress (diabetes-nonspecific and diabetes-specific) and diabetic complications in individuals with diabetes. The first reported that diabetes-nonspecific distress measured by the Mental Health Inventory (MHI-5) was associated with a higher risk of mortality (1.8-fold) and of having a cardiovascular event (1.7-fold) during the mean follow-up of 5.4 years in 1533 Swedish patients with type 2 diabetes [49]. The second study examined the prospective association between diabetes distress measured by PAID and future risk of diabetic complications in the UK [48]. This study revealed that diabetes distress (PAID score $\geq 40$ ) was not associated with macroand microvascular complications during the 2 year follow-up in participants with type 2 diabetes. However, the follow-up period of this study was too short to reveal the effect of diabetes distress on complications. The results of our study are valuable because we have revealed that diabetes distress was associated with high mortality risk even after adjusting for possible factors relevant to mortality risk such as glycaemic control or diabetic complications.

There were some limitations in the present study. First, because this is an epidemiological study, residual confounders may exist for the association of diabetes distress or depressive symptoms with all-cause mortality risk. Second, the data on the socioeconomic factors of participants were insufficient; therefore, it is yet to be elucidated whether this factor influences the association between diabetes distress or depressive symptoms and all-cause mortality risk. Third, we used CES-D to measure depressive symptoms, and this cannot be used to diagnose clinical depression; thus our study did not reveal the association among diabetes distress, depression and mortality risk. Nevertheless, it is meaningful that we have shown that the measurement of depressive symptoms using CES-D has predictive validity in terms of all-cause mortality risk. Finally, the data were obtained from the registry of a single diabetes centre, thereby raising concerns regarding generalisations derived from the results (e.g. primary care setting, region, ethnicity).

Both diabetes distress and depressive symptoms were independently associated with all-cause mortality risk in male participants with type 2 diabetes, a finding that was not observed in women. We did not observe significant interaction between diabetes distress and depressive symptoms.

Acknowledgements We would especially like to thank Y. Moritsuji, Y. Fujita, N. Nakamura and Y. Sakamoto (Department of Endocrinology, Tenri Hospital, Tenri, Nara, Japan) for their clerical support. The authors would like to thank Enago (www.enago.jp) for the English language review.

Data availability The data that support the findings of this study are available on request from the corresponding author. The data are not publicly available, because they contain information that could compromise research participant privacy. 
Funding This study was partially supported by the Manpei Suzuki Diabetes Foundation and Japan Society for the Promotion of Science (JSPS) KAKENHI (grant numbers: 25460641; 16 K08897; $19 \mathrm{~K} 10497)$. These organisations played no role in the study design or conduct, data collection, analysis or interpretation, and the preparation, review or approval of the manuscript.

Authors' relationships and activities $\mathrm{YH}$ reports personal fees from Boehringer Ingelheim, Merck \& Co., Inc., Kowa Pharmaceuticals, Inc., Astellas Pharma Inc., Ono Pharmaceutical Co., Ltd., Daiichi Sankyo Company, Ltd., Sumitomo Dainippon Pharma Co., Ltd., Takeda Pharmaceutical Company, Ltd. and Teijin Pharma Ltd., outside the submitted work. SO declares no relationships or activities that might bias, or be perceived to bias, their work. ST reports personal fees from AstraZeneca K.K., Daiichi Sankyo Company, Ltd., Eli Lilly Japan K.K., Mitsubishi Tanabe Pharma Corporation, Novo Nordisk Pharma Ltd., Ono Pharmaceutical Co., Ltd. and Sanofi K.K., outside the submitted work. HI reports personal fees from Takeda Pharmaceutical Company, Ltd., Eli Lilly Japan K.K., Sanofi KK., Merck \& Co., Inc., Astellas Pharma Inc., Novartis Pharma K.K., Mitsubishi Tanabe Pharma Corporation, Daiichi Sankyo Company, Ltd., Ono Pharmaceutical Co., Ltd., AstraZeneca K.K., Taisho Toyama Pharmaceutical Co., Ltd., Shionogi \& Co., Ltd., Kowa Pharmaceutical Co., Ltd. and Boehringer Ingelheim, outside the submitted work.

Contribution statement $\mathrm{YH}$ and $\mathrm{SO}$ searched the literature, conceived the study, analysed the data, interpreted the results, wrote the first draft of most sections of the report, obtained funding, collected the data, revised the report and participated in the writing of the report. YH was project coordinator. YH, SO, ST and HI organised and supervised the study, interpreted the results and revised the report. All authors finally approved the version to be published. YH is the guarantor of this work and, as such, had full access to all the data in the study and takes responsibility for the integrity of the data and the accuracy of the data analysis.

\section{Appendix}

Members of the Diabetes Distress and Care Registry in Tenri Study Group Hitoshi Ishii and Hirohito Kuwata (Department of Diabetology, Nara Medical University, Kashihara, Nara, Japan); Satoru Tsujii, Shintaro Okamura, Yasuaki Hayashino, Kentaro Kurosawa, Kiyoko Takano, Masaki Fujimura, Yui Sakuramachi and Masako Kitatani (Department of Endocrinology, Tenri Hospital, Tenri, Nara, Japan); Satoshi Matsunaga (Department of Hematology, Endocrinology and Metabolism, Niigata University Faculty of Medicine, Niigata, Japan); Yaeko Kondo and Naotaka Fujita (Department of Diabetes and Clinical Nutrition, Kyoto University, Kyoto, Japan); Rei Ueda (Second Department of Internal Medicine, Faculty of Medicine, University of the Ryukyus, Naha, Okinawa, Japan); Rie Kurokawa (Sawa Hospital, Osaka, Japan); Masami Tanaka (Division of Endocrinology, Metabolism and Nephrology, Department of Internal Medicine, Keio University School of Medicine, Tokyo, Japan); Tsuyoshi Mashitani (Mashitani Clinic, Kashihara, Japan); and Miyuki Furuya (Furuya Clinic, Nara, Japan).

\section{References}

1. Kahn SE, Cooper ME, Del Prato S (2014) Pathophysiology and treatment of type 2 diabetes: perspectives on the past, present, and future. Lancet 383:1068-1083. https://doi.org/10.1016/S01406736(13)62154-6

2. Whiting DR, Guariguata L, Weil C, Shaw J (2011) IDF diabetes atlas: global estimates of the prevalence of diabetes for 2011 and
2030. Diabetes Res Clin Pract 94:311-321. https://doi.org/10.1016/ j.diabres.2011.10.029

3. International Diabetes Federation (2017) IDF diabetes atlas, 8th edn. International Diabetes Federation, Brussels

4. Perrin NE, Davies MJ, Robertson N, Snoek FJ, Khunti K (2017) The prevalence of diabetes-specific emotional distress in people with type 2 diabetes: a systematic review and meta-analysis. Diabet Med 34:1508-1520. https://doi.org/10. 1111/dme. 13448

5. Ascher-Svanum H, Zagar A, Jiang D et al (2015) Associations between glycemic control, depressed mood, clinical depression, and diabetes distress before and after insulin initiation: an exploratory, post hoc analysis. Diabetes Ther 6:303-316. https://doi.org/10.1007/s13300015-0118-y

6. Dirmaier J, Watzke B, Koch U et al (2010) Diabetes in primary care: prospective associations between depression, nonadherence and glycemic control. Psychother Psychosom 79:172-178. https:// doi.org/10.1159/000296135

7. Egede LE, Osborn CY (2010) Role of motivation in the relationship between depression, self-care, and glycemic control in adults with type 2 diabetes. Diabetes Educ 36:276-283. https://doi.org/10. $1177 / 0145721710361389$

8. McKellar JD, Humphreys K, Piette JD (2004) Depression increases diabetes symptoms by complicating patients' self-care adherence. Diabetes Educ 30:485-492

9. Mashitani T, Hayashino Y, Okamura S et al (2015) Diabetes treatment-related quality of life is associated with levels of selfcare activities in insulin injection among Japanese patients with type 2 diabetes: Diabetes Distress and Care Registry at Tenri (DDCRT 8). Acta Diabetol 52:639-647. https://doi.org/10.1007/ s00592-015-0725-0

10. Snoek FJ, Bremmer MA, Hermanns N (2015) Constructs of depression and distress in diabetes: time for an appraisal. Lancet Diabetes Endocrinol 3:450-460. https://doi.org/10.1016/S2213-8587(15) 00135-7

11. Fisher L, Skaff MM, Mullan JT, Arean P, Glasgow R, Masharani U (2008) A longitudinal study of affective and anxiety disorders, depressive affect and diabetes distress in adults with type 2 diabetes. Diabet Med 25:1096-1101. https://doi.org/10.1111/j.1464-5491. 2008.02533.x

12. Albertorio-Diaz JR, Eberhardt MS, Oquendo M et al (2017) Depressive states among adults with diabetes: findings from the National Health and Nutrition Examination Survey, 2007-2012. Diabetes Res Clin Pract 127:80-88. https://doi.org/10.1016/j. diabres.2017.02.031

13. Tsujii S, Hayashino Y, Ishii H, Diabetes Distress and Care Registry at Tenri Study Group (2012) Diabetes distress, but not depressive symptoms, is associated with glycaemic control among Japanese patients with type 2 diabetes: Diabetes Distress and Care Registry at Tenri (DDCRT 1). Diabet Med 29:1451-1455. https://doi.org/10. 1111/j.1464-5491.2012.03647.x

14. Hayashino Y, Okamura S, Matsunaga S, Tsujii S, Ishii H, Tenri Cohort Study Group (2012) The association between problem areas in diabetes scale scores and glycemic control is modified by types of diabetes therapy: Diabetes Distress and Care Registry in Tenri (DDCRT 2). Diabetes Res Clin Pract 97:405-410. https://doi.org/ 10.1016/j.diabres.2012.04.005

15. Roy M, Sengupta N, Sahana PK et al (2018) Type 2 diabetes and influence of diabetes-specific distress on depression. Diabetes Res Clin Pract 143:194-198. https://doi.org/10.1016/j.diabres.2018.07. 006

16. Snoek FJ, Pouwer F, Welch GW, Polonsky WH (2000) Diabetesrelated emotional distress in Dutch and U.S. diabetic patients: crosscultural validity of the problem areas in diabetes scale. Diabetes Care 23:1305-1309. https://doi.org/10.2337/diacare.23.9.1305 
17. Snoek FJ, Kersch NY, Eldrup E et al (2011) Monitoring of Individual Needs in Diabetes (MIND): baseline data from the Cross-National Diabetes Attitudes, Wishes, and Needs (DAWN) MIND study. Diabetes Care 34:601-603. https://doi.org/10.2337/ dc10-1552

18. de Groot M, Anderson R, Freedland KE, Clouse RE, Lustman PJ (2001) Association of depression and diabetes complications: a meta-analysis. Psychosom Med 63:619-630. https://doi.org/10. 1097/00006842-200107000-00015

19. Hayashino $\mathrm{Y}$, Okamura S, Tsujii S, Ishii H, Diabetes Distress and Care Registry at Tenri Study Group (2018) Association between diabetes distress and all-cause mortality in Japanese individuals with type 2 diabetes: a prospective cohort study (Diabetes Distress and Care Registry in Tenri [DDCRT 18]). Diabetologia 61:1978-1984. https://doi.org/ 10.1007/s00125-018-4657-4

20. Ossip-Klein D, Rothenberg B, Andresen E (1997) Screening for depression assessing the health status of older adults. Springer, New York, pp 180-244

21. Randloff LS (1977) The CES-D scale: a self-report depression scale for research in the general population. Appl Psychol Meas 3:385401

22. Shima S, Shikano T, Kitamura T, Asai M (1985) Novel self-rating scale to evaluate depression. Clin Psychiatry 717-723 [article in Japanese]

23. Hayashino Y, Mashitani T, Tsujii S, Ishii H (2014) Serum highsensitivity C-reactive protein levels are associated with high risk of development, not progression, of diabetic nephropathy among Japanese type 2 diabetic patients: a prospective cohort study (Diabetes Distress and Care Registry at Tenri [DDCRT7]). Diabetes Care 37:2947-2952

24. Hayashino Y, Mashitani T, Tsujii S, Ishii H, Diabetes Distress and Care Registry at Tenri Study Group (2014) Elevated levels of hsCRP are associated with high prevalence of depression in Japanese patients with type 2 diabetes: the Diabetes Distress and Care Registry at Tenri (DDCRT 6). Diabetes Care 37:2459-2465. https://doi.org/10.2337/dc13-2312

25. Mashitani T, Hayashino $\mathrm{Y}$, Okamura $\mathrm{S}$, Tsujii $\mathrm{S}$, Ishii $\mathrm{H}$ (2014) Correlations between serum bilirubin levels and diabetic nephropathy progression among Japanese type 2 diabetic patients: a prospective cohort study (Diabetes Distress and Care Registry at Tenri [DDCRT 5]). Diabetes Care 37:252-258

26. Committee of the Japan Diabetes Society on the Diagnostic Criteria of Diabetes Mellitus; Seino Y, Nanjo K, Tajima N et al (2010) Report of the committee on the classification and diagnostic criteria of diabetes mellitus. J Diabetes Investig 1:212-228

27. Ishii H (2000) Psycho-behavioral problems in diabetes treatment. J Jpn Diabetes Soc 43:13-16 [article in Japanese]

28. Polonsky WH, Anderson BJ, Lohrer PA et al (1995) Assessment of diabetes-related distress. Diabetes Care 18:754-760. https://doi.org/ 10.2337/diacare.18.6.754

29. Welch GW, Jacobson AM, Polonsky WH (1997) The Problem Areas in Diabetes Scale. An evaluation of its clinical utility. Diabetes Care 20:760-766. https://doi.org/10.2337/diacare.20.5. 760

30. McHale M, Hendrikz J, Dann F, Kenardy J (2008) Screening for depression in patients with diabetes mellitus. Psychosom Med 70: 869-874. https://doi.org/10.1097/PSY.0b013e318186dea9

31. Buse JB, Bigger JT, Byington RP et al (2007) Action to Control Cardiovascular Risk in Diabetes (ACCORD) trial: design and methods. Am J Cardiol 99:21i-33i

32. Nakamura J, Kamiya H, Haneda M et al (2017) Causes of death in Japanese patients with diabetes based on the results of a survey of 45,708 cases during 2001-2010: Report of the Committee on
Causes of Death in Diabetes Mellitus. J Diabetes Investig 8:397410. https://doi.org/10.1111/jdi.12645

33. Stoop CH, Nefs G, Pop VJ et al (2014) Diabetes-specific emotional distress in people with type 2 diabetes: a comparison between primary and secondary care. Diabet Med 31:1252-1259. https:// doi.org/10.1111/dme.12472

34. Strandberg RB, Graue $M$, Wentzel-Larsen $T$, Peyrot $M$, Rokne B (2014) Relationships of diabetes-specific emotional distress, depression, anxiety, and overall well-being with HbA1c in adult persons with type 1 diabetes. J Psychosom Res 77:174-179. https://doi.org/10.1016/j.jpsychores.2014. 06.015

35. van Bastelaar KM, Pouwer F, Geelhoed-Duijvestijn PH et al (2010) Diabetes-specific emotional distress mediates the association between depressive symptoms and glycaemic control in type 1 and type 2 diabetes. Diabet Med 27:798-803. https://doi.org/10. 1111/j.1464-5491.2010.03025.x

36. Higginson IJ, Carr AJ (2001) Measuring quality of life: Using quality of life measures in the clinical setting. BMJ 322:1297-1300. https://doi.org/10.1136/bmj.322.7297.1297

37. Black SA, Markides KS, Ray LA (2003) Depression predicts increased incidence of adverse health outcomes in older Mexican Americans with type 2 diabetes. Diabetes Care 26:2822-2828. https://doi.org/10.2337/diacare.26.10.2822

38. Laake JP, Stahl D, Amiel SA et al (2014) The association between depressive symptoms and systemic inflammation in people with type 2 diabetes: findings from the South London Diabetes Study. Diabetes Care 37:2186-2192. https://doi.org/ $10.2337 / \mathrm{dc} 13-2522$

39. Zhang X, Norris SL, Gregg EW, Cheng YJ, Beckles G, Kahn HS (2005) Depressive symptoms and mortality among persons with and without diabetes. Am J Epidemiol 161:652-660. https://doi. org/10.1093/aje/kwi089

40. Gonzalez JS, Fisher L, Polonsky WH (2011) Depression in diabetes: have we been missing something important? Diabetes Care 34: 236-239

41. Cuijpers P, Vogelzangs N, Twisk J, Kleiboer A, Li J, Penninx BW (2014) Is excess mortality higher in depressed men than in depressed women? A meta-analytic comparison. J Affect Disord 161:47-54. https://doi.org/10.1016/j.jad.2014.03.003

42. Ariyo AA, Haan M, Tangen CM et al (2000) Depressive symptoms and risks of coronary heart disease and mortality in elderly Americans. Cardiovascular Health Study Collaborative Research Group. Circulation 102:1773-1779. https://doi.org/10.1161/01. CIR.102.15.1773

43. Das-Munshi J, Chang CK, Schofield P, Stewart R, Prince MJ (2019) Depression and cause-specific mortality in an ethnically diverse cohort from the UK: 8-year prospective study. Psychol Med 49:1639-1651

44. Oquendo MA, Ellis SP, Greenwald S, Malone KM, Weissman MM, Mann JJ (2001) Ethnic and sex differences in suicide rates relative to major depression in the United States. Am J Psychiatry 158:1652-1658. https://doi.org/10.1176/appi.ajp.158.10.1652

45. Robins LN, Helzer JE, Croughan J, Ratcliff KS (1981) National Institute of Mental Health Diagnostic Interview Schedule. Its history, characteristics, and validity. Arch Gen Psychiatry 38:381-389. https://doi.org/10.1001/archpsyc.1981.01780290015001

46. Fisher L, Glasgow RE, Strycker LA (2010) The relationship between diabetes distress and clinical depression with glycemic control among patients with type 2 diabetes. Diabetes Care 33: 1034-1036. https://doi.org/10.2337/dc09-2175

47. Pintaudi B, Lucisano G, Gentile S et al (2015) Correlates of diabetes-related distress in type 2 diabetes: Findings from the benchmarking network for clinical and humanistic outcomes in diabetes (BENCH-D) study. J Psychosom Res 79:348-354. https://doi.org/10.1016/j.jpsychores.2015.08.010 
48. Ismail K, Moulton CD, Winkley K et al (2017) The association of depressive symptoms and diabetes distress with glycaemic control and diabetes complications over 2 years in newly diagnosed type 2 diabetes: a prospective cohort study. Diabetologia 60:2092-2102. https://doi.org/10.1007/s00125-017-4367-3

49. Dalsgaard EM, Vestergaard M, Skriver MV et al (2014) Psychological distress, cardiovascular complications and mortality among people with screen-detected type 2 diabetes: follow-up of the ADDITION-Denmark trial. Diabetologia 57:710-717. https:// doi.org/10.1007/s00125-014-3165-4

Publisher's note Springer Nature remains neutral with regard to jurisdictional claims in published maps and institutional affiliations. 